Rev. Psicol. (Arequipa. Univ. Catól. San Pablo) / Año 2019 / Vol 9 / N 3 / pp. 37-55

ISSN 2306-0565 versión impresa / ISSN 2311-7397 versión on line

(c) $(\$) \begin{aligned} & \text { Esta obra está bajo una Licencia Creative Commons } \\ & \text { Atribución 4.o Internacional (BY-NC-ND) }\end{aligned}$

\title{
CLIMA ORGANIZACIONAL Y RENDIMIENTO LABORAL EN SERVIDORES ADMINISTRATIVOS DE UNA UNIVERSIDAD PÚBLICA DE LA CIUDAD DE AREQUIPA
}

\author{
ORGANIZATIONAL CLIMATE AND LABOUR PERFORMANCE AMONG \\ ADMINISTRATIVE PERSONNEL FROM A PUBLIC UNIVERSITY IN AREQUIPA
}

\author{
José Manuel Calizaya López y Rildo Santos Bellido Medina \\ Universidad Nacional de San Agustín, Arequipa, Perú
}

\begin{abstract}
Resumen
El presente estudio pretende valorar el clima organizacional y el rendimiento laboral entre los trabajadores administrativos de una universidad pública de Arequipa. Se evaluó a 339 trabajadores de entre 20 y 70 años, siendo el 43.4\% mujeres y el 56.6\% varones. Se aplicó la Escala de Clima Organizacional y el Cuestionario de Rendimiento Laboral. El análisis estadístico reveló que ni el sexo y ni la edad, se asocian con el clima organizacional o el rendimiento laboral. Sin embargo, el clima organizacional y el rendimiento laboral mostraron diferencias significativas en función del cargo. Finalmente, el clima organizacional y el rendimiento laboral solo obtuvieron correlaciones nulas.
\end{abstract}

Palabras claves: Clima organizacional, rendimiento laboral, psicología organizacional.

\begin{abstract}
This study was designed to assess the organizational climate and job performance among administrative workers at a public university in Arequipa. . We tested 339 workers between 20 and 70 years of age (43.4\% female and 56.6\% male). We administered the Organizational Climate Scale and the Labour Performance Questionnaire. The statistical analysis revealed that neither gender nor age, were associated with organizational climate or labour performance,
\end{abstract}


although, the organizational climate and labour performance showed significant differences according to the participants' positions in the University. Finally, the association between organizational climate and labour performance only obtained null correlations.

Keywords: Organizational climate, labour performance, organizational psychology.

\section{Introducción}

Según Ivancevich (2006), el clima organizacional estudia los comportamientos, actitudes y desempeño humano en una empresa; basándose en teorías, métodos y principios de la psicología, la sociología y la antropología cultural; para entender la manera en cómo se percibe, se valora las capacidades de aprendizaje y las acciones individuales del trabajador. Luthans (2008) por otra parte, manifiesta que el clima organizacional es la aplicación de las fortalezas y las capacidades psicológicas del área de recursos humanos, que se miden, sedesarrollan y seadministran eficazmente para el mejoramiento del desempeño en el lugar del trabajo actual. Sin embargo, hay un consenso entre las interpretaciones más conocidas sobre el clima organizacional (Arias, 2013), que lo refiere a la manera cómo perciben los trabajadores de una organización el trabajo, el ambiente, el ambiente físico en que éste se da, las relaciones interpersonales que tienen lugar en torno a él y las diversas regulaciones formales que afectan a dicho trabajo.

Por otro lado, los factores que determinan en el clima laboral son los siguientes:

a. Factores externos. Los factores externos de la organización como los clientes, proveedores, presiones sindicales y los medios de comunicación.

b. Factores psicológicos. Losfactores psicológicosqueestán presentes comolasactitudes, las opiniones, las percepciones, las motivaciones y las expectativas de las personas durante sus interacciones.

c. Factores individuales, grupales y estructurales. Las características de cada individuo, comprende sus actitudes, percepciones, personalidad, valores, motivaciones y el nivel de estrés que puedan sentir influye sobre su comportamiento y sobre o ambiente al interior de la organización.

El clima organizacional resulta entonces, de la forma cómo las personas establecen procesos de interacción social (Méndez, 2006) y se ve influenciado por un sistema de valores, actitudes y creencias, como también por el ambiente o entorno. De hecho, un estudio local reciente, reveló que el clima organizacional tiene un impacto positivo y significativo en las relaciones interpersonales de los trabajadores de una empresa del sector privado (Arias, Lazo \& Quintana, 2018).

En ese sentido, según Martínez (2003) el clima organizacional determina la forma en que los individuos perciben el ambiente 
de trabajo, su rendimiento personal, su nivel de productividad y el grado de satisfacción que experimentan siendo parte de la organización en la cual labora. Por ello, nos basamos en un modelo desarrollado por Calizaya, Bellido y Huamani (2019) que sugiere que el clima organizacional se compone de tres dimensiones: cultura organizacional, diseño organizacional y potencial humano.

Se entiende como cultura organizacional la forma característica de pensar y hacer las cosas en una organización, producto de las interacciones para adaptarse a circunstancias del entorno y a tensiones internas (Schein, 1996). Existen 10 características primarias que concentran la esencia de la cultura organizacional:

1. La identidad de sus miembros: el grado en que los empleados se identifican con la organización como un todo y no solo con su tipo de trabajo o campo de conocimientos.

2. Énfasis en el grupo: las actividades laborales se organizan en torno a grupos y no a personas.

3. El enfoque hacia las personas: las decisiones de la administración toman en cuenta las repercusiones que los resultados tendrán en los miembros de la organización.

4. La integración de unidades: la forma como se fomenta que las unidades de la organización funcionen de forma coordinada e independiente.
5. El control: el uso de reglamentos procesos y supervisión directa para controlar la conducta de los individuos.

6. Tolerancia al riesgo: el grado en que se fomenta que los empleados sean agresivos, innovadores y arriesgados.

7. Los criterios para recompensar: como se distribuyen las recompensas, como los aumentos de sueldo y los ascensos, de acuerdo con el rendimiento del empleado y por su antigüedad, favoritismo $\mathrm{u}$ otros factores ajenos al rendimiento.

8. El perfil hacia los fines o los medios: de qué manera la administración se perfila hacia los resultados o metas y no hacia las técnicas o procesos usados para alcanzarlos.

9. El enfoque hacia un sistema abierto: el grado en que la organización controla y responde a los cambios externos.

10.Tolerancia al conflicto: grado en que la organización fomenta que los miembros traten abiertamente sus conflictos y críticas (Schein, 1996).

La cultura organizacional es un sistema particular de símbolos, influido por la sociedad circundante, por la historia de la organización y por sus líderes pasados, así como también por diferentes factores de contingencia. Según esta concepción, la cultura no es un elemento estático, sino una materia prima viviente utilizada de manera diferente por cada empleado y 
transformada por ellos durante el proceso de decodificación de los acontecimientos organizacionales. De esta manera, la cultura organizacional es una herramienta eficaz para la interpretación de la vida y del comportamiento organizacionales y para la comprensión de los procesos de decadencia, adaptación y cambio radical en las organizaciones (Allaire y Firsirotu, 1992). Según el modelo que aquí se trabaja, la cultura organizacional se compone de siete indicadores: Motivación, identidad, apoyo, equidad, trabajo en equipo, organización y condiciones de trabajo.

Así tenemos que la motivación es para Robbins (2008) la disposición para hacer algo, en donde la habilidad de la persona para satisfacer alguna necesidad condicionada de ese algo. La identidad organizacional está constituida por valores y creencias, considerándose a las creencias como convicciones compartidas y la idea generalizada de lo que es importante, y los valores son los estándares establecidos y aceptados por el grupo sobre lo que son y sus formas de reaccionar ante los imprevistos (Deal y Kennedy, 1999). El apoyo es una manifestación tanto individual como grupal o institucional que favorece los procesos de afrontamiento en el trabajo (Arias, 2013). La equidad se refiere a la igualdad de oportunidades que tienen los trabajadores de lograr sus metas laborales y de acceder a promociones en el trabajo. El trabajo en equipo es la ejecución de un proyecto a cargo de un grupo de personas, cuyo elemento clave es la capacidad de autogestión, es decir que, el grupo de personas están capacitadas para realizar las acciones que demanda la empresa (Mapcal, 1998). La organización es la coordinación de actividades diferentes, de colaboradores individuales, para llevar a cabo transacciones planeadas con el ambiente (Lawrence y Lorsch, 1973), y las condiciones laborales de un trabajador son los factores de su entorno intrínseco, que determinan la motivación, la satisfacción o insatisfacción laboral; y como menciona Chiavenato (2009), la motivación de las personas para el trabajo depende de dos factores íntimamente relacionados: factores higiénicos y factores motivacionales.

Por otro lado, según Hellrieguel y Slocum (2014), el diseño organizacional es el proceso de elegir una estructura de tareas, responsabilidades y relaciones de autoridad dentro de las organizaciones, que representa las conexiones entre varias divisiones o departamentos de una organización en un organigrama. Según nuestro modelo, esta dimensión se compone de tres componentes: comunicación, reconocimiento y espacio físico.

En cuanto a la primera, Katz y Kahn (1977) definen a la comunicación como el intercambio de información y difusión de significados. La comunicación fluye horizontal (entre posiciones de un mismo rango) o verticalmente (a nivel jerárquico); y se transmite de diversas formas (escritos, verbal, etc.). Su contenido es diverso (ordenes, informes, quejas, etc.) y tiene un costo económico. También se puede diferenciar entre comunicación formal e informal. En la primera incluyen todos los canales y medios de comunicación 
que en la organización se establecen de manera consciente y deliberada. Abarca comunicaciones verbales, memorandas, cartas, actas, informes o manuales oficiales. Por su parte, la comunicación informal comprende aquel conjunto de mensajes no escritos o rumores que se intercambian como resultado de la amistad o compañerismo (Simón, 1988).

Por reconocimiento se entiende la percepción que tienen los trabajadores sobre la retribución que reciben por el esfuerzo realizado, y constituye el primer incentivo en una relación laboral. No solo se refiere al establecimiento de un salario justo, sino de incentivos adicionales (no necesariamente monetarios) que motiven al trabajador a realizar un mejor desempeño (Litwin y Stringer, 1978). La OMS (2010), define como ambiente físico del trabajo a la parte de los recursos del espacio de trabajo que puede detectarse mediante monitoreos humanos o electrónicos e incluyen la estructura, aire, maquinaria, equipo, productos, químicos, materiales y procesos que se realizan o están presentes en el espacio de trabajo; y que pueden afectar la seguridad física o mental, la salud y el bienestar de los trabajadores. En ese sentido, Mejía (2011), refiere que los diversos factores ambientales pueden generar un gran impacto en la vida de los individuos, e incidir en diferentes aspectos, desde el comportamiento general hasta en la salud física, psicológica y social de los individuos.
En cuanto al potencial humano, este constructo abarca una diversidad de variables propias del trabajador que se alinean con la gestión del talento (Chiavenato, 2002). Dentro de estas capacidades tenemos la innovación, que describe el proceso a través del cual las ideas creativas se desarrollan en algo tangible, como por ejemplo un nuevo producto o práctica (Henry, 1991). El liderazgo es la habilidad de influir positivamente en la gente y los sistemas provocando una actitud determinada, bajo la autoridad de una persona a fin de tener un impacto significativo y lograr resultados importantes (Evans y Lindsay, 2008). La autonomía, es entendida como la capacidad que tienen los profesionales de controlar o manejar de una forma eficaz su situación laboral (Ruíz, 2015), y la cohesión, que según Balaguer, Castillo, Moreno, Garrigues y Soriano (2004), es una variable que ayuda a entender la dinámica del grupo y a desarrollar el buen clima en un equipo de trabajo.

Como segunda variable de este estudio tenemos al rendimiento laboral, que es definido por Chiavenato (2000) como las acciones o comportamientos observados en los empleados que son relevantes en el logro de los objetivos de la organización. Bittel (2000) plantea que el desempeño es influenciado en gran parte por las expectativas del empleado sobre el trabajo, sus actitudes hacia los logros y su deseo de armonía. Por tanto, el desempeño se relaciona o vincula con las habilidades y conocimientos que apoyan las acciones del trabajador, en pro de consolidar los objetivos de la empresa. Ghiselli (1998) 
señala como el desempeño está influenciado por cuatro factores: la motivación, habilidades y rasgos personales, claridad y aceptación del rol, y oportunidades para realizarse. La importancia de este enfoque reside en el hecho que el desempeño del trabajador va de la mano con las actitudes y aptitudes que estos tengan en función a los objetivos que se quieran alcanzar, seguidos por políticas normas, visión y misión de la organización.

De acuerdo con nuestro modelo propuesto (Calizaya, Bellido, Arias \& Huamani, 2019) el rendimiento laboral se compone de seis factores: asistencia y puntualidad, actitud hacia el trabajo, desempeño laboral, trabajo en equipo, orientación hacia los resultados y planificación. La puntualidad es una actitud que se adquiere desde los primeros años de vida mediante la formación de hábitos en la familia, donde las normas y costumbres establecen horarios para cada una de nuestras actividades (Orbegoso, 2007). Por lo que, la puntualidad es una disciplina, que se adquiere con la práctica y con el sacrificio según el temperamento de cada persona (Waichman, 2003).

Robbins (2008) define actitud, "como los juicios evaluativos, favorables o desfavorables, sobre objetos, personas o acontecimientos. Manifiestan la opinión de quienes hablan acerca de algo" (p. 235). El autor indica que las actitudes no se asemejan con los valores, pero sí se encuentran relacionadas; así, la actitud se encuentra compuesta por tres componentes: cognición, afecto y comportamiento. Así mismo, Robbins (2008) indica que la persona puede poseer muchas actitudes, pero centrándonos en el comportamiento organizacional son tres: satisfacción con el trabajo, participación en el trabajo y compromiso con la organización.

Robbins y Coulter (2010) entienden que el rendimiento laboral es un proceso para determinar qué tan exitosa ha sido una organización (o un individuo o un proceso) en el logro de sus actividades y objetivos laborales. En general a nivel organizacional la medición del desempeño laboral brinda una evaluación acerca del cumplimento de las metas estratégicas a nivel individual. Así también, Robbins y Judge (2013) explican que, en las organizaciones, solo se evalúan la forma en que los empleados realizan sus actividades y estas incluyen una descripción del puesto de trabajo, sin embargo, las compañías actuales, menos jerárquicas y más orientadas al servicio, requieren de más información. Hoy en día se reconocen tres tipos principales de conductas que constituyen el desempeño laboral, como son el desempeño de las tareas, el civismo y falta de productividad.

En cuanto al trabajo en equipo como dimensión del rendimiento laboral, Posner (200o), lo define como una modalidad de articular las actividades laborales de un grupo humano en torno a un conjunto de fines, de metas y de resultados a alcanzar. El trabajo en equipo implica una interdependencia 
activa entre los integrantes de un grupo que comparten y asumen una misión de trabajo. Para Robbins (2008), la velocidad y la capacidad de reacción ante determinadas circunstancias son los dos elementos principales de la orientación a resultados, una característica que tienen las organizaciones para acercarse a los objetivos propuestos de la forma más eficaz posible. Especialistas de la gestión empresarial como Robbins (2008), creen incluso, que es una competencia estrechamente vinculada a la capacidad de adaptación de las instituciones a su entorno, sobre todo si se tiene en cuenta que el mercado actual cambia continuamente y exige a los profesionales de esta área nuevas competencias y habilidades.

Según Chiavenato (2000), a planificación es la primera función administrativa porque sirve de base para las demás funciones. Esta función determina por anticipado cuáles son los objetivos que deben cumplirse y qué debe hacerse para alcanzarlos; por tanto, es un modelo teórico para actuar en el futuro. La planificación comienza por establecer los objetivos y detallar los planes necesarios para alcanzarlos de la mejor manera posible. La planificación determina donde se pretende llegar, qué debe hacerse, cómo, cuándo y en qué orden.

En vista de estas definiciones conceptuales y operativas, el fin de este estudio es valorar las relaciones entre el clima organizacional y el rendimiento laboral, según los modelos propuestos, analizando también de manera comparativa, cómo se manifiestan estas variables en función del sexo, la condición laboral y el cargo de los trabajadores de una universidad pública de la ciudad de Arequipa.

\section{Método}

\section{Participantes}

El estudio se realizó con servidores administrativos de una universidad pública de la ciudad de Arequipa. La muestra fue aleatoria y estuvo conformada por 339 servidores de una población total de 1479 trabajadores. La edad media de los servidores administrativos fue de 43 años con una desviación estándar de \pm 11.63 , en un rango de 20 a 70 años. El $43.4 \%$ fueron mujeres y el $56.6 \%$ varones. La condición laboral fue del 57.2\% nombrados, el 34.5 CAS (Contrato Administrativo de Servicios) y el 8.3\% con contrato a tiempo fijo; de los cuales el 40,1\% trabajan como secretarias, el $13.9 \%$ conserje, el $11.2 \%$ asistente, el $9.4 \%$ vigilante, el $8.6 \%$ bibliotecario, el $6.8 \%$ técnicos y el restante $10 \%$ trabajan como jardinero, almacenista, cajero, nutricionista, reportero y camarógrafo.

\section{Instrumentos}

Se utilizó la Escala del Clima Organizacional elaborada y validada por Calizaya, Bellido y Huamani (2019), el cual consta de 50 ítems de respuesta tipo Likert ( $1=$ muy en desacuerdo, hasta, $5=$ muy de acuerdo) que evalúa tres dimensiones: La primera dimensión es la Cultura organizacional con siete indicadores, que son Motivación (ítems: 
1, 2, 3, 4), Identidad (Ítems: 5, 6, 7), Apoyo (Îtems: 8, 9, 10), Equidad (Ítems: 11, 12, 13, 14), Trabajo en equipo (Ítems: 15, 16, 17), Organización (Îtems:18, 19, 20) y Condiciones de trabajo (Îtems: 21, 22, 23, 24). La segunda dimensión es el Diseño Organizacional con tres indicadores, que son la Comunicación organizacional (Îtems: 25, 26, 27, 28), Reconocimiento (Îtems: 29, 30, 31, 32) y Espacio físico (Îtems: 33, 34, 35, 36) y la tercera dimensión Potencial Humano con cuatro indicadores que son la Innovación (Îtems: 37, 38, 39, 40), Liderazgo (Ítems: 41, 42, 43), Autonomía $(44,45,46)$ y Cohesión $(47,48,49,50)$. Las mismas que presentan confiabilidad alta (Cultura organizacional $\omega=$ .95; Diseño organizacional $\omega=.86$ y Potencial Humano $\omega=.91$ ).

El otro instrumento utilizado fue el Cuestionario de Rendimiento Laboral, elaborado y validado por Calizaya, Bellido, Arias y Huamani (2019), el mismo que presenta 6 factores y 47 ítems, Asistencia y puntualidad con 4 ítems (1, 2, 3,4); Actitud hacia el trabajo con 11 ítems $(5,6,7,8,9,10,11,12,13,14$, 15); Desempeño laboral con 7 ítems (16, $17,18,19,20,21,22)$; Trabajo en equipo con 11 ítems $(23,24,25,26,27,28,29$, 30, 31, 32, 33); Orientación a resultados con 8 ítems $(34,35,36,37,38,39,40,41)$ y Planificación con 6 ítems (42, 43, 44, 45, 46, 47). Se obtuvieron los niveles de confiabilidad de los 6 factores mediante el método de consistencia interna y con la prueba alfa de Cronbach: .32 para Asistencia y puntualidad, .96 para Actitud hacia el trabajo, .78 para
Desempeño laboral, .8o para Trabajo en equipo, .93 para Orientación a resultados y .92 para el factor Planificación. En los instrumentos se adjuntaron preguntas para medir características sociodemográficas como edad, sexo, condición laboral, cargo y área laboral; y se consignaron los datos propios del consentimiento informado.

\section{Procedimiento}

La Escala de Clima Organizacional fue aplicada a los servidores administrativos en su área de trabajo, y el cuestionario de Rendimiento laboral fue aplicado al jefe inmediato superior de cada uno de los servidores administrativos seleccionados en la muestra. La evaluación fue hecha de acuerdo al cargo laboral y al área de trabajo. Esta evaluación se realizó con la autorización y en coordinación con las autoridades de la universidad, y previo consentimiento informado y reserva total de la información individual. Para el análisis de la información se utilizó el programa estadístico SPSS versión 24. Se exploró la distribución de los datos, y se evidenció que no existe normalidad ( $\mathrm{p}<.05)$, por lo tanto, el análisis estadístico utilizado fue no paramétrico. Se realizó el análisis descriptivo y el comparativo de las variables y sus dimensiones según edad, sexo, condición laboral y cargo. La comparación de dos muestras independientes se utilizó con el estadístico U de MannWhitney, la comparación de más de dos muestras independientes (comparando los puntajes del rango promedio) con la $\mathrm{H}$ de Kruskal-Wallis y las correlaciones 
con la Rho de Spearman. Asimismo, se aplicó el calculó de la probabilidad de superioridad (PSest) como tamaño del efecto para U de Mann-Whitney, las normas interpretativas son: No efecto (PSest $\leq$ o.o); pequeño (PSest $\geq 0,56)$; mediano (PSest $\geq 0.64$ ) y grande (PSest $\geq$ o.71) (Grissom, 1994). Para calcular el tamaño del efecto de Kruskal-Wallis se utilizó el coeficiente épsilon al cuadrado (ER2), (Tomczak \& Tomczak, 2014), las normas interpretativas son: pequeño ( $E R 2 \geq 0.01)$; mediano $(E R 2 \geq 0.06)$ y grande $\left(E R_{2} \geq 0.14\right)$ (Faul, Erdfelder, Lang, \& Buchner, 2007).

\section{Resultados}

En la Tabla 1, se observa con respecto a la cultura organizacional, que el $50 \%$ de los investigados presenta puntajes menores a 80 , de un puntaje máximo de 115 , que indica un puntaje medio con tendencia al puntaje alto. En el diseño organizacional menos del $50 \%$ de participantes presenta 38 puntos de un rango de 12 a 50 puntos, que indica un nivel medio. En la dimensión potencial humano se observa que el $50 \%$ de los participantes, presenta puntajes menores a 45 de un rango de 14 a 70 , que indica un puntaje medio con tendencia al puntaje alto. Finalmente, en la variable clima organizacional el $50 \%$ de participantes presenta puntajes menores a 162 de un rango de 50 a 245 puntos, que indican un nivel medio.

Tabla 1. Valores descriptivos del clima organizacional

\begin{tabular}{|lccccc|}
\hline & $\boldsymbol{M}$ & $\boldsymbol{D E}$ & $\boldsymbol{M d}$ & Min. & Max. \\
\hline Cultura Organizacional & 79.2 & 16.3 & 80.0 & 24 & 115 \\
Diseño organizacional & 37.9 & 9.2 & 38.0 & 12 & 60 \\
Potencial Humano & 45.7 & 10.2 & 45.0 & 14 & 70 \\
Clima organizacional & 162.9 & 33.6 & 162.0 & 50 & 245 \\
\hline
\end{tabular}

Nota: $M=$ media aritmética; $D E=$ desviación estándar; $M d=$ mediana; $M i n=$ puntaje mínimo; Max= puntaje máximo

No existen diferencias estadísticamente es decir, los valores de probabilidad de significativas del clima organizacional y superioridad (tamaño del efecto), se aprosus dimensiones según sexo (Tabla 2), ximan a un efecto pequeño (PSest< $<.43$ ). 
Tabla 2. Análisis comparativo de las dimensiones del clima organizacional según sexo

\begin{tabular}{|c|c|c|c|c|c|}
\hline Dimensiones & $\begin{array}{c}\text { Hombres } \\
(n=192) \\
\text { Mdn (Ric') }\end{array}$ & $\begin{array}{c}\text { Mujeres } \\
(n=147) \\
\text { Mdn (Ric') }\end{array}$ & $U$ & $p$ & $P S_{\text {est }}$ \\
\hline Cultura Organizacional & $78.0(22.0)$ & $81.0(23.0)$ & 13444.5 & 0.455 & 0.48 \\
\hline Diseño organizacional & $38.0(13.7)$ & $38.0(14.0)$ & 13916.5 & 0.827 & 0.49 \\
\hline Potencial Humano & $45.0(15.0)$ & $45.0(15.0)$ & 13558.5 & 0.536 & 0.48 \\
\hline Clima organizacional & $158.0(50.5)$ & $163.0(47.0)$ & 13538.0 & 0.521 & 0.48 \\
\hline
\end{tabular}

Nota: $n=$ tamaño muestral; $U=U$ de Mann-Whitney; $p=$ p valor; $P S e s t=$ Probabilidad de Superioridad (tamaño del efecto)

En la Tabla 3, se observa que no existen diferencias estadísticamente significativas del clima organizacional y sus dimensiones según condición laboral, es decir, los valores de coeficiente épsilon al cuadrado (tamaño del efecto), se aproximan a un efecto pequeño $(E R 2=0.003)$.

Tabla 3. Análisis comparativo de las dimensiones del clima organizacional según condición laboral

\begin{tabular}{|c|c|c|c|c|c|c|}
\hline Dimensiones & $\begin{array}{l}\text { Nombrado } \\
\text { (n=194) } \\
\text { Mdn (Ric') }\end{array}$ & $\begin{array}{c}\text { Contrato } \\
\text { plazo fijo } \\
(n=28) \\
\operatorname{Mdn}\left(\text { Ric }^{\prime}\right)\end{array}$ & $\begin{array}{c}\text { CAS } \\
(n=117) \\
\operatorname{Mdn}\left(\operatorname{Ric}^{\prime}\right)\end{array}$ & $\boldsymbol{H}$ & $p$ & $E_{R}^{2}$ \\
\hline Cultura organizacional & $79.5(22.2)$ & $81.5(28.0)$ & $81.0(23.0)$ & 0.4 & 0.823 & 0.001 \\
\hline Diseño organizacional & $37.5(13.0)$ & $37.5(14.2)$ & $39.0(14.0)$ & 1.3 & 0.515 & 0.003 \\
\hline Potencial humano & $45.0(13.2)$ & $46.5(19.7)$ & $45.0(15.0)$ & 0.1 & 0.970 & 0.001 \\
\hline Clima organizacional & $158.5(48.2)$ & $165.0(56.7)$ & $162.0(52.0)$ & 0.3 & 0.851 & 0.001 \\
\hline
\end{tabular}

Nota: $\boldsymbol{n}=$ tamaño muestral; $\boldsymbol{H}=\mathrm{H}$ de Kruskal Wallis; $\boldsymbol{p}=\mathrm{p}$ valor; $E R \mathbf{R}=$ coeficiente épsilon al cuadrado (tamaño del efecto)

En la Tabla 4, se observa que existen diferencias en las dimensiones diseño organizacional, potencial humano y la variable clima organizacional según cargo laboral, donde el coeficiente $(E R 2=.04)$ indica que la proporción de variabilidad de estas dimensiones y de la variable pueden atribuirse al cargo laboral (efecto pequeño). En la dimensión cultura organizacional, no existen diferencias significativas y el coeficiente $(E R 2=0.03)$ indica un efecto pequeño. De modo que, al analizar los rangos promedio, se observa que los participantes con el cargo de conserje, secretaria, asistente, son los que presentan puntajes más altos, a diferencia de los participantes con el cargo de bibliotecario y vigilante que presentan puntajes bajos. 
Tabla 4. Análisis comparativo de las dimensiones y del clima organizacional según cargo

\begin{tabular}{|c|c|c|c|c|c|c|c|c|c|c|}
\hline Dim. & $\begin{array}{c}\text { Bibliotecario } \\
(n=29) \\
\text { Mdn }\left(\text { Ric }^{\prime}\right)\end{array}$ & $\begin{array}{c}\text { Conserje } \\
(n=47) \\
M d n\left(\text { Ric }^{\prime}\right)\end{array}$ & $\begin{array}{c}\text { Secretaria } \\
(n=136) \\
\operatorname{Mdn}\left(\text { Ric }^{\prime}\right)\end{array}$ & $\begin{array}{c}\text { Asistente } \\
(n=38) \\
\text { Mdn (Ric') }\end{array}$ & $\begin{array}{c}\text { Vigilante } \\
(n=32) \\
\text { Mdn }\left(\text { Ric }^{\prime}\right)\end{array}$ & $\begin{array}{c}\text { Técnico } \\
(n=23) \\
\text { Mdn (Ric') }\end{array}$ & $\begin{array}{c}\text { Otros } \\
(n=34) \\
\text { Mdn (Ric') }\end{array}$ & $H$ & $p$ & $E_{R}^{2}$ \\
\hline 1 & $75.0(27.5)$ & $81.0(23.0)$ & $81.0(22.5)$ & $81.0(22.2)$ & $74.0(33.0)$ & 76.0 (10.0) & 84.5 (34.0) & 10.8 & 0.09 & 0.03 \\
\hline 2 & $38.0(14.5)$ & 39.0 (12.0) & $38.0(12.0)$ & $39.5(10.2)$ & $31.5(17.7)$ & $35.0(13.0)$ & $40.5(17.2)$ & 12.8 & 0.04 & 0.04 \\
\hline 3 & $43.0(18.5)$ & 47.0 (14.0) & $46.0(15.0)$ & $45.0(12.7)$ & $39.5(19.2)$ & $40.0(9.0)$ & $46.0(15.2)$ & 12.3 & 0.05 & 0.04 \\
\hline 4 & $155.0(57.5)$ & $164.0(43.0)$ & $164.5(47.2)$ & $165.5(40.0)$ & $141.0(70.5)$ & 149.0 (30.0) & $170.0(62.0)$ & 13 & 0.04 & 0.04 \\
\hline
\end{tabular}

Nota: $\boldsymbol{n}=$ tamaño muestral; $\boldsymbol{H}=\mathbf{H}$ de Kruskal Wallis; $\boldsymbol{p}=\mathbf{p}$ valor; $\boldsymbol{E R} \mathbf{2}=$ coeficiente épsilon al cuadrado (tamaño del efecto); $1=$ Cultura organizacional; $2=$ Diseño organizacional; $3=$ Potencial humano; $4=$ Clima organizacional.

A nivel correlacional, se observa que no existe relación entre la edad, la variable clima organizacional y sus dimensiones; sin embargo, las tres dimensiones del clima organizacional, cultura organizacional, diseño organizacional y potencial humano están correlacionadas de manera fuertey positiva con la puntuación global del clima organizacional, y de manera moderada entre sí (ver Tabla 5).

Tabla 5. Correlaciones del clima organizacional y sus dimensiones con la edad

\begin{tabular}{|lccccc|}
\hline & 1 & 2 & 3 & 4 & 5 \\
\hline Edad & 1.000 & & & & \\
Cultura Organizacional & 0.031 & 1.000 & & & \\
Diseño organizacional & 0.015 & $.842^{* *}$ & 1.000 & & \\
Potencial Humano & 0.009 & $.804^{* *}$ & $.784^{* *}$ & 1.000 & \\
Clima organizacional & 0.026 & $.960^{* *}$ & $.926^{* *}$ & $.908^{* *}$ & 1.000 \\
\hline
\end{tabular}

En la Tabla 6, se observa con respecto al rendimiento laboral, que el 50\% de los servidores administrativos presenta puntajes menores a 109, de un rango de 58 a 199, que indica un puntaje medio con tendencia baja. Con respecto a las dimensiones: actitud hacia el trabajo $(M d=25)$, desempeño laboral $(M d=$ $15)$, orientación a resultados $(M d=15)$ y planificación $(M d=13)$ indican un puntaje medio con tendencia baja. Sin embargo, la dimensión trabajo en equipo $(M d=28)$ indican un puntaje medio, a diferencia de la dimensión asistencia y puntualidad, donde el 50\% de participantes presenta puntajes menores a 14 de un rango de 5 a 20 puntos, que indica un puntaje medio con tendencia al puntaje alto. 
Tabla 6. Valores descriptivos del rendimiento laboral

\begin{tabular}{|lccccc|}
\hline & $\boldsymbol{M}$ & $\boldsymbol{D E}$ & $\boldsymbol{M d}$ & $\boldsymbol{M i n}$. & Max. \\
\hline Asistencia y puntualidad & 14.2 & 2.4 & 14.0 & 5 & 20 \\
Actitud hacia el trabajo & 25.6 & 10.2 & 25.0 & 12 & 55 \\
Desempeño laboral & 15.4 & 4.6 & 15.0 & 7 & 33 \\
Trabajo en equipo & 27.4 & 6.7 & 28.0 & 11 & 48 \\
Orientación a resultados & 16.0 & 6.2 & 15.0 & 8 & 35 \\
Planificación & 13.3 & 5.1 & 13.0 & 6 & 30 \\
Rendimiento Laboral & 111.9 & 29.5 & 109.0 & 58 & 199 \\
\hline
\end{tabular}

Nota: $M=$ media aritmética; $D E=$ desviación estándar; $M d=$ mediana; $M i n=$ puntaje mínimo; Max= puntaje máximo

No existen diferencias estadísticamente superioridad (tamaño del efecto), se aprosignificativas del rendimiento laboral y ximan a un efecto pequeño (PSest $<0.49$ ), sus dimensiones según sexo (Tabla 7), que indican que el rendimiento laboral es decir, los valores de probabilidad de no diferencia entre hombres y mujeres.

Tabla 7. Análisis de las diferencias de del rendimiento laboral según sexo

\begin{tabular}{|c|c|c|c|c|c|}
\hline Dimensiones & $\begin{array}{c}\text { Hombres } \\
(n=192) \\
\operatorname{Mdn}\left(\text { Ric }^{\prime}\right)\end{array}$ & $\begin{array}{c}\text { Mujeres } \\
(n=147) \\
\text { Mdn (Ric') }\end{array}$ & $U$ & $p$ & $P S_{\text {est }}$ \\
\hline Asistencia y puntualidad & $15.0(3.0)$ & $14.0(3.0)$ & 13591.5 & 0.555 & 0.48 \\
\hline Actitud hacia el trabajo & $24.5(20.0)$ & $25.0(13.0)$ & 13995.0 & 0.896 & 0.49 \\
\hline Desempeño laboral & $15.0(6.7)$ & $15.0(6.0)$ & 13731.0 & 0.669 & 0.48 \\
\hline Trabajo en equipo & $27.0(11.0)$ & $28.0(7.0)$ & 12975.0 & 0.203 & 0.45 \\
\hline Orientación a resultados & $15.0(10.0)$ & $16.0(7.0)$ & 13675.5 & 0.625 & 0.48 \\
\hline Planificación & $13.0(9.0)$ & $12.0(6.0)$ & 13505.0 & 0.496 & 0.47 \\
\hline Rendimiento Laboral & $109.0(50.5)$ & $110.0(34.0)$ & 13740.5 & 0.678 & 0.48 \\
\hline
\end{tabular}

Nota: $n=$ tamaño muestral; $U=U$ de Mann-Whitney; $p=$ p valor; $P S e s t=$ Probabilidad de Superioridad (tamaño del efecto)

En la Tabla 8, se observa que existen diferencias en las dimensiones actitud hacia el trabajo, desempeño laboral, trabajo en equipo, orientación a resultados, planificación y la variable rendimiento laboral, donde el coeficiente $\left(E R_{2}<0.04\right)$ indica que la proporción de variabilidad de estas dimensiones y de la variable pueden atribuirse al cargo laboral (efecto pequeño), donde los servidores administrativos nombrados y CAS son los que presentan puntajes más altos que los servidores por contrato a plazo fijo; los mismos que en la dimensión asistencia y puntualidad 
presentan puntajes más altos, con un coeficiente $(E R 2=0.02)$ que indica un efecto pequeño.

Tabla 8. Análisis comparativo de las dimensiones del rendimiento laboral según condición laboral

\begin{tabular}{|c|c|c|c|c|c|c|}
\hline Dimensiones & $\begin{array}{l}\text { Nombrado } \\
(n=194) \\
\operatorname{Mdn}\left(\text { Ric }^{\prime}\right)\end{array}$ & $\begin{array}{c}\text { Contrato } \\
\text { plazo fijo } \\
(n=28) \\
\operatorname{Mdn}\left(\text { Ric }^{\prime}\right)\end{array}$ & $\begin{array}{c}\text { CAS } \\
(n=117) \\
\operatorname{Mdn}\left(\text { Ric }^{\prime}\right)\end{array}$ & $\boldsymbol{H}$ & $p$ & $E_{R}^{2}$ \\
\hline Asistencia y puntualidad & $14.0(3.0)$ & $15.0(2.0)$ & $14.0(3.0)$ & 6.4 & 0.04 & 0.02 \\
\hline Actitud hacia el trabajo & $24.0(17.2)$ & $17.5(12.5)$ & $26.0(12.5)$ & 8.7 & 0.013 & 0.03 \\
\hline Desempeño laboral & $15.0(6.0)$ & $12.5(4.7)$ & $16.0(6.0)$ & 7.2 & 0.028 & 0.02 \\
\hline Trabajo en equipo & $27.0(11.0)$ & $24.5(5.0)$ & $28.0(7.5)$ & 6.5 & 0.038 & 0.02 \\
\hline Orientación a resultados & $14.0(8.0)$ & $13.0(8.7)$ & $17.0(7.0)$ & 8.1 & 0.017 & 0.02 \\
\hline Planificación & $12.0(8.0)$ & $11.0(6.0)$ & $14.0(7.0)$ & $7 \cdot 7$ & 0.021 & 0.02 \\
\hline Rendimiento Laboral & $107.5(45.0)$ & $92.5(32.7)$ & $114.0(38.5)$ & 8.5 & 0.014 & 0.03 \\
\hline
\end{tabular}

Nota: $n=$ tamaño muestral; $H=H$ de Kruskal Wallis; $p=$ p valor; $E R 2=$ coeficiente épsilon al cuadrado (tamaño del efecto)

En la Tabla 9, se observa que existen diferencias en las dimensiones, desempeño laboral, trabajo en equipo, orientación a resultados, planificación y la variable rendimiento laboral, donde el coeficiente $(E R 2<0.04)$ indica que la proporción de variabilidad de estas dimensiones y de la variable pueden atribuirse al cargo laboral (efecto pequeño), donde los servidores administrativos con cargo de conserjey secretaria son los que presentan puntajes más altos que los otros servidores. Asimismo, en la dimensión actitud hacia el trabajo, los servidores con cargo de conserje y secretaria presentan puntajes más altos, con un coeficiente $(E R 2=$ o.o6) que indica un efecto mediano. Sin embargo, en la dimensión asistencia y puntualidad los servidores con cargo de vigilante presentan puntajes más altos que los otros servidores con un coeficiente $(E R 2=.06)$ que indica un efecto mediano. 
Tabla 9. Análisis comparativo de las dimensiones del rendimiento laboral según cargo

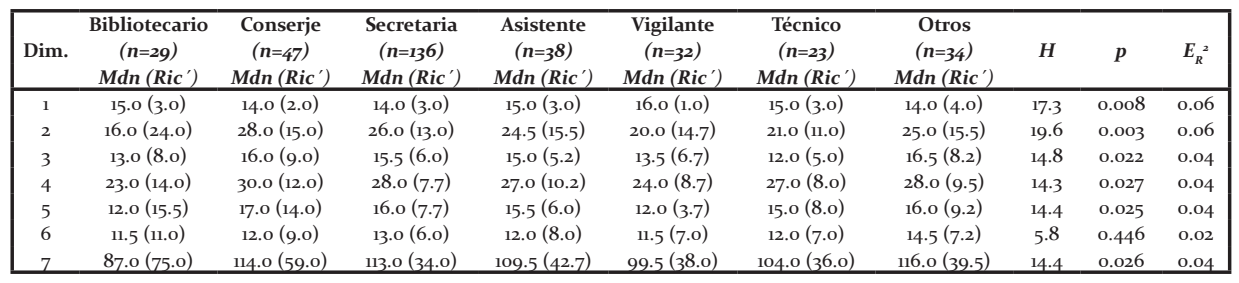

Nota: $n=$ tamaño muestral; $H=H$ de Kruskal Wallis; $p=p$ valor; $E R 2=$ coeficiente épsilon al cuadrado (tamaño del efecto); 1=Asistencia y puntualidad; $2=$ Actitud hacia el trabajo; $3=$ Desempeño laboral; $4=$ Trabajo en equipo; $5=$ Orientación a resultados; $6=$ Planificación; $7=$ Rendimiento laboral

A nivel correlacional, se observa que existe relación entre la edad y algunas dimensiones del rendimiento laboral, estas relaciones son bajas y negativas, es decir, a mayor edad menor actitud hacia el trabajo, menor desempeño laboral, menos trabajo en equipo, menor orientación a resultados. En general a mayor edad menor rendimiento laboral yviceversa. Sin embargo, las dimensiones que no se relacionan con la edad son la asistenciay puntualidady la planificación. Además, se observa que las dimensiones correlacionan entre sí, donde la dimensión asistencia y puntualidad presenta correlación negativa de nivel medio con todas las otras dimensiones y la variable rendimiento laboral. Las otras dimensiones están correlacionadas de manera fuertey positiva con la puntuación global del rendimiento laboral (ver Tabla 10).

Tabla 10. Correlaciones del rendimiento laboral y sus dimensiones con la edad

\begin{tabular}{|llccccccc|}
\hline & Edad & $\mathbf{1}$ & $\mathbf{2}$ & $\mathbf{3}$ & $\mathbf{4}$ & $\mathbf{5}$ & $\mathbf{6}$ & 7 \\
\hline Edad & 1.000 & & & & & & & \\
1. Asistencia y puntualidad & 0.065 & 1.000 & & & & & & \\
2. Actitud hacia el trabajo &,$- 155^{* *}$ &,$- 484^{* * *}$ & 1.000 & & & & & \\
3. Desempeño laboral &,$- 120^{*}$ &,$- 453^{* *}$ &, $822^{* *}$ & 1.000 & & & & \\
4. Trabajo en equipo &,$- 149^{* * *}$ &,$- 481^{* *}$ &, $857^{* *}$ &, $800^{* *}$ & 1.000 & & & \\
5. Orientación a resultados &,$- 143^{* *}$ &,$- 462^{* *}$ &, $791^{* *}$ &, $748^{* *}$ &, $797^{* *}$ & 1.000 & \\
6. Planificación & -0.101 &,$- 458^{* *}$ &, $841^{* *}$ &, $793^{* *}$ &, $821^{* *}$ &, $819^{* *}$ & 1.000 & \\
7. Rendimiento Laboral &,$- 158^{* *}$ &,$- 457^{* *}$ &, $950^{* *}$ &, $886^{* *}$ &, $930^{* *}$ &, $891^{* *}$ &, $918^{* *}$ & 1.000 \\
\hline
\end{tabular}

La Tabla 11, muestra que no existe relación entre el clima organizacional y sus dimensiones con el rendimiento laboraly sus dimensiones, es decir, el rendimiento laboral no es posible explicarlo a través del clima organizacional en los servidores administrativos de la universidad investigada. 
Tabla 11. Correlaciones del clima organizacional y el rendimiento laboral

\begin{tabular}{|lcccc|}
\hline & $\begin{array}{c}\text { Cultura } \\
\text { organizacional }\end{array}$ & $\begin{array}{c}\text { Diseño } \\
\text { organizacional }\end{array}$ & $\begin{array}{c}\text { Potencial } \\
\text { humano }\end{array}$ & $\begin{array}{c}\text { Clima } \\
\text { organizacional }\end{array}$ \\
\hline 1. Asistencia y puntualidad & -0.067 & -0.096 & -0.083 & -0.077 \\
2. Actitud hacia el trabajo & -0.007 & 0.014 & -0.036 & -0.007 \\
3. Desempeño laboral & 0.035 & 0.031 & -0.038 & 0.021 \\
4. Trabajo en equipo & -0.042 & -0.004 & -0.07 & -0.043 \\
5. Orientación a resultados & -0.021 & 0.002 & -0.093 & -0.025 \\
6. Planificación & -0.002 & 0.007 & -0.072 & -0.013 \\
7. Rendimiento Laboral & -0.011 & 0.007 & -0.069 & -0.018 \\
\hline
\end{tabular}

\section{Discusión}

Para Chiavenato (2000) el clima organizacional constituye el medio interno de una organización, donde la atmósfera en la que se constituye es peculiar a cada organización. Asimismo, menciona que el concepto de clima organizacional involucra diferentes aspectos de la situación, que se sobreponen mutuamente en diversos grados, como el tipo de organización, la tecnología, las políticas, las metas operacionales, los reglamentos internos (factores estructurales); además de las actitudes, sistemas de valores y formas de comportamiento social que son impulsadas o castigadas (factores sociales).

Estos indicadores que se mencionan tienen relación con el tipo de organización que hoy en día presenta la Universidad Nacional de San Agustín de Arequipa, y con los cambios que generan en ella, a través de la implementación de la nueva ley universitaria $\left(\mathrm{N}^{\circ}\right.$ 30220), cuya finalidad es mejorar y fortalecer las universidades peruanas. En ese sentido, los resultados de la presente investigación indican que el clima organizacional que perciben los servidores administrativos, evidencian que un $61.4 \%$ consideran que este se encuentra en un nivel medio, con una tendencia a ser alto en un 31.3\% y percibido como bajo en un $7 \cdot 4 \%$.

El análisis inferencial en función de tres variables sociodemográficas revela ciertos aspectos que merecen ser comentados, pues el sexo de los trabajadores no es una variable que evidencie diferencias en el clima organizacional, así como tampoco existe una correlación significativa entre el clima organizacional o sus dimensiones y la edad de los trabajadores evaluados. Tampoco se registraron diferencias significativas entre los trabajadores de esta universidad pública, en función de la condición laboral, sin embargo, el cargo de los trabajadores sí presenta diferencias estadísticamente significativas en las dimensiones de diseño organizacional y potencial humano, así como en el clima organizacional como constructo global. Aunque los tamaños del efecto son bajos, se puede apreciar que los trabajadores en el cargo de conserje, secretaria y asistente, 
son los que presentan puntajes más altos, mientras que los trabajadores con cargo de bibliotecario y vigilante presentan los puntajes más bajos.

Por otro lado, el rendimiento laboral tiene a ser bajo entre los trabajadores de la universidad pública de Arequipa, y tampoco se evidencian diferencias estadísticamente significativas entre los puntajes de los trabajadores en función del sexo, y se registran correlaciones muy bajas entre la edad con el rendimiento laboral y sus respectivas dimensiones. No obstante, la condición laboral y el cargo de los trabajadores sí registra diferencias estadísticamente significativas en los puntajes del rendimiento laboral (y sus dimensiones). Así, se tiene que el personal administrativo nombrado y CAS presentan puntajes más altos aquellos trabajadores que tienen contrato a plazo fijo, los mismos que en la dimensión asistencia y puntualidad presentan puntajes más altos, pero con tamaños del efecto pequeños.
Por otro lado, en cuanto al cargo, los trabajadores administrativos con cargo de conserje y secretaria son los que presentan puntajes más altos que sus compañeros. Asimismo, en la dimensión actitud hacia el trabajo, los servidores con cargo de conserje y secretaria presentan puntajes más altos, con tamaño del efecto mediano. Sin embargo, en la dimensión asistencia y puntualidad los trabajadores con cargo de vigilante presentan puntajes más altos que los otros colaboradores, también con un tamaño del efecto mediano.

Finalmente, las correlaciones entre el clima organizacional y el rendimiento laboral, cada una con sus respectivas dimensiones, arrojó coeficientes de correlación nulos que sugieren que ambas variables se conducen de manera independiente; es decir, que no existe relación entre el rendimiento laboral y el clima organizacional, al menos en los servidores administrativos de esta universidad pública, ubicada en el sur del Perú. 


\section{Referencias}

Allaire, Y., \& Firsirotu, M. (1992). Cultura Organizacional:Aspectos teóricos, prácticos y metodológicos. Bogotá, Colombia: Legis.

Arias, W. L. (2013). Clima organizacional en ocho empresas de Arequipa. Illustro, $4,39-56$.

Arias, W. L., Lazo, J., \& Quintana, S. (2018). ¿Es el clima organizacional determinante de las relaciones interpersonales o son las relaciones interpersonales las que determinan el clima organizacional? Industrial data, 21(2), 81-90.

Balaguer, I., Castillo, I., Moreno, Y., Garrigues, V., \& Soriano, L. (2004). El clima motivacional y la cohesión en equipos de fútbol. Encuentros en Psicología Social, 2(1), 152-156.

Bittel, L. (200o). Administración de Personal. México: Pearson Educación

Calizaya, J., Bellido, R., Arias, W. L., \& Huamani, J. C. (2019). Construcción y validación de una escala de rendimiento laboral en la ciudad de Arequipa. Manuscrito sometido a revisión.

Calizaya, J., Bellido, R., \& Huamani, J. C. (2019). Construcción y validación de la Escala del Clima Organizacional en la ciudad de Arequipa. Manuscrito sometido a revisión.

Chiavenato, I. (2000). Administración de Recursos Humanos. Colombia: McGraw-Hill.

Chiavenato, I. (2002). Gestión del talento humano. Bogotá, Colombia: McGraw-Hill.

Chiavenato, I. (2009). Comportamiento Organizacional. México: McGraw-Hill.

Deal, T., \& Kennedy, A. (1999). The New Corporate Cultures. Massachusetts, USA: Perseus Books Reading.

Evans, J., \& Lindsay, W. (2008). Administración y control de calidad. México, D.F: Cengage Learning.

Faul, F., Erdfelder, E., Lang, A.G., \& Buchner, A. (2007). G*Power 3: A flexible statistical power analysis program for the social, behavioral, and biomedical sciences. Behavior Research Methods, 39(2), 175-191. 
Ghiselli, J. (1998). Administración de personal. Colombia: s/e.

Grissom, R.J. (1994). Probability of the superior outcome of one treatment over another. Journal of Applied Psychology, 79, 314-16.

Hellriegel, D. y Slocum J. (2014). Comportamiento Organizacional. 1oma Edición. México: Thomson.

Henry, J. (1991). Make sense of creativity. En J. Henry (Ed.), Creative Management. Londres: Sage Publications.

Ivancevich, M. (2006). Comportamiento Organizacional. México: McGraw-Hill.

Katz, D., \& Kahn, R. (1977). Psicología Social de las Organizaciones. México, Trillas.

Lawrence, P., \& Lorsch, J. (1973). Desarrollo de organizaciones: diagnóstico y acción. USA: Fondo Educativo Interactivo.

Litwin, G., \& Stringer, R. (1978). Organizational Climate. New York: Simon \& Schuster.

Luthans, F. (2008). Comportamiento organizacional. México: McGraw-Hill.

Mapcal, A. (1998). Gestión eficaz del trabajo en equipo. Madrid: Díaz de Santos

Martínez, M. (2003). La gestión empresarial: equilibrando objetivos y valores. Madrid: Díaz de Santos.

Mejía, A. (2011). Estrés ambiental e impacto de los factores ambientales en la escuela. Pampedia, 7, 3-18.

Organización Mundial de la Salud (OMS). (2010). Entornos laborales saludables. Fundamentos y Modelo de la OMS. Contextualización, Prácticas y Literatura de Soporte. Recuperado de: http://www.istas.ccoo.es/descargas/guia\%2ode\%20 sensibilizacion\%2odef.pdf

Orbegoso, A. (2007). La deshora peruana o la impuntualidad en el Perú. Colección Realidad Nacional, Vol. 7. Lima: Universidad Ricardo Palma.

Posner, P. (200o). Trabajo en equipo. Buenos Aires: IIPE.

Robbins S. (2008). Comportamiento organizacional. México: Pearson educación. 
Robbins, S., \& Coulter, M. (2010). Administración. México, D.F.: Pearson Educación.

Robbins, S., \& Judge, T. (2013). Comportamiento Organizacional. México: Pearson Educación.

Ruíz, A. (2015). Sobrecarga laboral y autonomía en trabajadores sociales en relación con el síndrome de burnout. (Trabajo de grado). Universidad de La Rioja, España.

Simón, H. (1988). El comportamiento administrativo. Argentina: Editorial Aguilar.

Tomczak, M., \& Tomczak, E. (2014). The need to report effect size estimates revisited. An overview of some recommended measures of effect size. Trends Sport Sciences, 1(21), 19-25.

Waichman, P. (2003). Tiempo libre y recreación. un desafío pedagógico. Buenos Aires: Ediciones PW.

Recibido: 2 de abril de 2019

Aceptado: 19 de agosto de 2019 